\title{
OPTIMUM PROBLEMS WITH CERTAIN LOWER SEMICONTINUOUS SET-VALUED CONSTRAINTS*
}

\author{
ZSOLT PÁLES ${ }^{\dagger}$ AND VERA ZEIDAN ${ }^{\ddagger}$
}

\begin{abstract}
In this paper, we provide a complete analysis of second-order admissible variations to inequality type constraints that are given in terms of lower semicontinuous set-valued functions whose images are closed convex sets with nonempty interior. As an application, we consider optimization problems where such constraints are present and we deduce second-order necessary conditions for optimality.
\end{abstract}

Key words. set-valued maps, lower semicontinuous, nonempty interior, convex sets in $C\left(T, \mathbf{R}^{r}\right)$, support functional, tangent and normal cones, second-order admissible variations

AMS subject classifications. Primary 90C48, 90C34; Secondary 58C06, 47H04

PII. S105262349630725X

1. Introduction. Consider the following optimization problem:

$$
(\mathcal{P}) \quad \text { Minimize } f(z) \text { subject to } G(z) \in \mathbf{Q}, H(z)=0 \text {, }
$$

where $f: D \rightarrow \mathbf{R}, G: D \rightarrow X, H: D \rightarrow Y, X, Y, Z$ are Banach spaces, $D \subset Z$ is nonempty and open, and $\mathbf{Q} \subset X$ is a closed convex set with nonempty interior.

The prototype of such problems arises, for instance, in optimal control theory with state constraints in the inclusion form $x(t) \in Q(t)(\forall t \in T)$, where $Q(\cdot)$ is a lower semicontinuous set-valued map with closed convex nonempty interior images.

Better understanding of optimality conditions is an ongoing research program for several researchers. This question is of great value in theory and in applications. As is widely known, such conditions must always be given in terms of the original data of the problem and, in the context of necessity, are expected to be as strong as they can be.

In 1988, Kawasaki [6], [8] discovered, for the problem $(\mathcal{P})$, where $\mathbf{Q}$ is a cone, second-order necessary conditions that contain an extra term manifesting the presence of infinitely many inequalities in the constraint $G(z) \in \mathbf{Q}$. This phenomenon is known as an "envelope-like effect." Such a result was generalized by Cominetti in [2]. Both results assumed a Mangasarian-Fromovitz-type condition and evoked in the extra term the second-order tangent set:

$$
T^{2}(x, d \mid \mathbf{Q})=\liminf _{t \rightarrow 0+} \frac{\mathbf{Q}-x-t d}{t^{2}} .
$$

In [11] the authors have generalized the previous results in [6], [8], and [2] to the nondifferentiable case without assuming a Mangasarian-Fromowitz condition. However, instead of $T^{2}(x, d \mid \mathbf{Q})$, they have used the second-order admissible variation set

* Received by the editors July 24, 1996; accepted for publication (in revised form) July 2, 1997; published electronically June 3, 1998.

http://www.siam.org/journals/siopt/8-3/30725.html

$\dagger$ Institute of Mathematics, L. Kossuth University, H-4010 Debrecen, Pf. 12, Hungary (pales@borel.math.klte.hu). The research of this author was supported by Hungarian National Foundation for Scientific Research (OTKA) grant T-016846.

$¥$ Department of Mathematics, Michigan State University, East Lansing, MI 48824 (zeidan@ math.msu.edu). The research of this author was supported by the National Science Foundation under grant DMS-9404591. 
$V(x, d \mid \mathbf{Q})$ whose elements are vectors $v \in X$ satisfying the following condition: there exists $\bar{\varepsilon}>0$ such that

$$
x+\varepsilon d+\varepsilon^{2}(v+w) \in \mathbf{Q} \quad \text { for all } \quad 0<\varepsilon<\bar{\varepsilon},\|w\|<\bar{\varepsilon}, w \in X .
$$

That is,

$$
V(x, d \mid \mathbf{Q})=\bigcup_{\bar{\varepsilon}>0} \bigcap_{\substack{\varepsilon<\bar{\varepsilon} \\\|w\|<\bar{\varepsilon}}}\left[\frac{1}{\varepsilon^{2}}(\mathbf{Q}-x-\varepsilon d)+w\right] .
$$

It follows directly from the definition that $V(x, d \mid \mathbf{Q})$ is an open convex set. Moreover, it is shown in [11] that

$$
\overline{V(x, d \mid \mathbf{Q})}=T^{2}(x, d \mid \mathbf{Q}) .
$$

The results obtained in [11] are analogous to those obtained by Maruyama [9, Theorem 3.2 , who uses a Neustadt derivative to handle the nonsmoothness of data.

In order to recall the first- and second-order necessary conditions for $(\mathcal{P})$, obtained in [11, Corollary 2], we need to introduce the following notation and notions.

A point $\hat{z} \in D$ is called an admissible point for $(\mathcal{P})$ if $G(\hat{z}) \in \mathbf{Q}$ and $H(\hat{z})=0$ hold.

A point $\hat{z} \in D$ is called a regular point for $(\mathcal{P})$ if

(R1) $f$ is locally Lipschitz at $\hat{z}$;

(R2) $G$ is strictly Fréchet differentiable at $\hat{z}$;

(R3) $H$ is strictly Fréchet differentiable at $\hat{z}$ and the range of the linear operator $H^{\prime}(\hat{z})$ is a closed subspace of $Y$.

If $f$ is locally Lipschitz at $\hat{z}$, then the expression

$$
f^{o}(\hat{z} ; d):=\limsup _{(z, \varepsilon) \rightarrow(\hat{z}, 0+)} \frac{f(z+\varepsilon d)-f(z)}{\varepsilon}
$$

is finite and will be called Clarke's generalized directional derivative. For properties, see [1].

Let $\hat{z}$ be an admissible regular point for $(\mathcal{P})$ and $d \in Z$. A vector $d \in Z$ is called a critical direction at $\hat{z}$ for $(\mathcal{P})$ if

(C1) $f^{o}(\hat{z} ; d) \leq 0$

(C2) $G^{\prime}(\hat{z}) d \in \overline{\text { cone }}(\mathbf{Q}-G(\hat{z}))=: T(G(\hat{z}) \mid \mathbf{Q})$;

(C3) $H^{\prime}(\hat{z}) d=0$.

A vector $d \in Z$ is called a regular direction at $\hat{z}$ for $(\mathcal{P})$ if the following hold.

(R4) $f^{o o}(\hat{z}, d):=\limsup _{\varepsilon \rightarrow 0+} 2 \frac{f(\hat{z}+\varepsilon d)-f(\hat{z})-\varepsilon f^{o}(\hat{z} ; d)}{\varepsilon^{2}}$ is finite.

(R5) The second-order directional derivatives

$$
G^{\prime \prime}(\hat{z}, d):=\lim _{\varepsilon \rightarrow 0+} 2 \frac{G(\hat{z}+\varepsilon d)-G(\hat{z})-\varepsilon G^{\prime}(\hat{z}) d}{\varepsilon^{2}}
$$

and

$$
H^{\prime \prime}(\hat{z}, d):=\lim _{\varepsilon \rightarrow 0+} 2 \frac{H(\hat{z}+\varepsilon d)-H(\hat{z})-\varepsilon H^{\prime}(\hat{z}) d}{\varepsilon^{2}}
$$

exist. 
(R6) The set of second-order admissible variations of $\mathbf{Q}$ at $G(\hat{z})$ in the direction $G^{\prime}(\hat{z}) d$ is not empty, i.e. $V\left(G(\hat{z}), G^{\prime}(\hat{z}) d \mid \mathbf{Q}\right) \neq \emptyset$.

Clearly, the zero vector is always a regular critical direction at $\hat{z}$ for $(\mathcal{P})$.

Now we are ready to state the result of [11, Corollary 2].

THEOREM 1.1. Let $\hat{z}$ be a regular solution of the above problem $(\mathcal{P})$. Then, for all regular critical directions $d$, there exist Lagrange multipliers $\lambda \geq 0, x^{*} \in X^{*}$, and $y^{*} \in Y^{*}$ such that at least one of them is different from zero and the following relations hold.

$$
\lambda f^{o}(\hat{z} ; z)+\left\langle x^{*}, G^{\prime}(\hat{z}) z\right\rangle+\left\langle y^{*}, H^{\prime}(\hat{z}) z\right\rangle \geq 0 \quad \text { for } \quad z \in Z
$$

and

$$
\lambda f^{o o}(\hat{z}, d)+\left\langle x^{*}, G^{\prime \prime}(\hat{z}, d)\right\rangle+\left\langle y^{*}, H^{\prime \prime}(\hat{z}, d)\right\rangle \geq 2 \delta^{*}\left(x^{*} \mid V\left(G(\hat{z}), G^{\prime}(\hat{z}) d \mid \mathbf{Q}\right)\right) .
$$

(Here $\delta^{*}$ stands for the support function [14].)

Results along the line of Theorem 1.1 were obtained by Ioffe [5] and Penot [13] for the differentiable case and in the presence of a certain qualification condition. In these works, the superior second-order tangent set is used, namely,

$$
\mathbf{Q}^{\prime \prime}(x, d):=\limsup _{t \rightarrow 0+} \frac{\mathbf{Q}-x-t d}{t^{2}} .
$$

Clearly, $\mathbf{Q}^{\prime \prime}(x, d)$ is larger than $T^{2}(x, d \mid \mathbf{Q})=\overline{V(x, d \mid \mathbf{Q})}$, but as noted in [7], this set is more difficult to compute and hence is not as useful in the applications.

The relationship between $V$ and $T^{2}$ shows that either of the two sets can be used in Theorem 1.1. Two important questions naturally arise from Theorem 1.1.

(i) How can we check the nonemptiness of $V(x, d \mid \mathbf{Q})$, since otherwise the secondorder optimality conditions would be satisfied trivially?

(ii) How do we evaluate the support function at $V(x, d \mid \mathbf{Q})$ and hence $V(x, d \mid \mathbf{Q})$ ?

In order that $V(x, d \mid \mathbf{Q})$ be nonempty, it is only necessary that $\mathbf{Q}$ have a nonempty interior and that $d$ belong to $\overline{c o n e}(\mathbf{Q}-x)=T(x \mid \mathbf{Q})$, which is the tangent cone to $\mathbf{Q}$ at $x$. If $d \in \operatorname{cone}(\mathbf{Q}-x)$, then $V(x, d \mid \mathbf{Q})$ is nonempty and $V(x, d \mid \mathbf{Q})=$ cone(cone(int $\mathbf{Q}-$ $x)-d)$ (cf. [11, Theorem 4]). In this case the extra term in the second-order condition (1.3) disappears. However, examples are provided by Kawasaki [6] in order to show that the necessary conditions with extra term, that is, when $d \in \overline{c o n e}(\mathbf{Q}-x)$, handle situations that cannot be handled with previous results where $d$ is taken from cone $(\mathbf{Q}-$ $x)$. Thus one has to also consider directions $d \in T(x \mid \mathbf{Q}) \backslash \operatorname{cone}(\mathbf{Q}-x)$. In this case the description of $V(x, d \mid \mathbf{Q})$ and its nonemptiness is far from being trivial.

The key role of the two questions stated above is also stressed by Kawasaki [7], to which Penot [13] also refers the readers. In the same paper [7], Kawasaki was able to answer these two questions pertaining to the set $T^{2}(x, d \mid \mathbf{Q})$ for only the special case when $\mathbf{Q}$ has the form

$$
\mathbf{Q}:=C_{+}(T):=\{x \in C(T, \mathbf{R}): x(t) \geq 0 \forall t \in T\},
$$

where $T$ is a compact metric space. These results, pertaining to the set $V$, were generalized in [11, Theorem 3] by the authors for the nondifferentiable setting. 
In the following theorem we recall the results of Kawasaki [7, Theorem 2.1], [8, Lemma 3.1].

Theorem 1.2. Let $x \in C_{+}(T)$ and $d \in \overline{\operatorname{cone}}\left(C_{+}(T)-x\right)$. Let $T_{0}$ be the set of $t_{0} \in T$ satisfying the following property: there exists a sequence $t_{n} \in T$ such that

$$
x\left(t_{n}\right)>0, \quad t_{n} \rightarrow t_{0}, \quad \text { and } \quad \frac{d\left(t_{n}\right)}{x\left(t_{n}\right)} \rightarrow-\infty \quad \text { as } n \rightarrow \infty .
$$

Define the function $\mathbf{E}=\mathbf{E}_{x, d}$ by

$$
\mathbf{E}(t):= \begin{cases}\sup \left\{\limsup _{n \rightarrow \infty} \frac{d\left(t_{n}\right)^{2}}{4 x\left(t_{n}\right)}:\left(t_{n}\right) \text { satisfies }(1.4)\right\} & \text { if } t \in T_{0}, \\ 0 & \text { if } x(t)=d(t)=0, t \notin T_{0}, \\ -\infty & \text { otherwise. }\end{cases}
$$

Then

(a) $T^{2}(x, d \mid \mathbf{Q}) \neq \emptyset$ if and only if $\mathbf{E}_{x, d}(t)<+\infty$ for all $t \in T$;

(b) a function $v \in C(T, \mathbf{R})$ belongs to $T^{2}\left(x, d \mid C_{+}(T)\right)$ if and only if $\mathbf{E}_{x, d}(t) \leq v(t)$ for all $t \in T$.

The function $\mathbf{E}$ defined in Theorem 1.2 can be expressed in another form; see Remark 3.3 below.

Of much more interest is the case when $\mathbf{Q}$ is a subset of $C\left(T, \mathbf{R}^{r}\right)$ defined by

$$
\mathbf{Q}=\left\{x(\cdot) \in C\left(T, \mathbf{R}^{r}\right) \mid x(t) \in Q(t) \forall t \in T\right\},
$$

where $Q(\cdot)$ is a lower semicontinuous set-valued map whose images are closed, convex, and have nonempty interior. The importance of this type of constraint stems from control problems with state constraints. Indeed, it is stated by Loewen and Rockafellar in [10]: "A satisfactory treatment of problems whose velocity constraint is described intrinsically by a differential inclusion is one of the principal accomplishments of nonsmooth analysis."

For the important case, when $\mathbf{Q}$ is given by (1.5), the two questions (i) and (ii) stated above are still open. In this case, the above question can be rephrased as follows:

$\left(^{*}\right)$ Characterize the nonemptiness and the support functional of $V(x, d \mid \mathbf{Q})$ in terms of the images $Q(t)$ and their support functionals $\delta^{*}(\cdot \mid Q(t))$.

Note that, by [12], the set $\mathbf{Q}$ defined by (1.5) is " $C$-convex" (see section 2) and thus $V(x, d \mid \mathbf{Q})$ is also "C-convex." Therefore, $V(x, d \mid \mathbf{Q})$ can be identified with a lower semicontinuous set-valued function $V(x, d \mid \mathbf{Q})(\cdot)$ whose images are nonempty convex open sets. In this case, by [15] and Theorem $2.1, \delta^{*}(\cdot \mid V(x, d \mid \mathbf{Q}))$ is expressed in terms of $\delta^{*}(\cdot \mid V(x, d \mid \mathbf{Q})(\cdot))$ and, by [12] and Corollary 2.3, the nonemptiness of $V(x, d \mid \mathbf{Q})$ is characterized by

$$
\delta^{*}(\xi \mid V(x, d \mid \mathbf{Q})(t))>-\infty \quad \forall(t, \xi) \in T \times \mathbf{R}^{r} .
$$

Furthermore, the correspondence $V(x, d \mid \mathbf{Q}) \rightarrow q(\cdot, \cdot)$ is bijective, where

$$
q(t, \xi):=\delta^{*}(\xi \mid V(x, d \mid \mathbf{Q})(t))
$$

is jointly lower semicontinuous and sublinear in $\xi$. Therefore, the questions in $\left(^{*}\right)$ are equivalent to finding for $q(\cdot, \cdot)$, defined in (1.7), an expression explicitly given in terms of $\delta^{*}(\cdot \mid Q(\cdot)), x(\cdot)$, and $d(\cdot)$. This task is far from trivial. 
The aim of this paper is to positively answer the open questions in $\left(^{*}\right)$ in such a way that extends the result known for the case where $\mathbf{Q}=C_{+}(T)$. In section 2 , we review recent results on convex analysis developed in [12] and needed for the rest of the paper. In section 3, we introduce a function $\sigma(x, d \mid Q)(t, \xi)$ in terms of $\delta^{*}(\xi \mid Q(t)), x(\cdot)$, and $d(\cdot)$. This function turns out to be jointly lower semicontinuous and positively homogeneous in $\xi$. In Theorem 3.2, we provide a characterization of $V(x, d \mid \mathbf{Q})$ in terms of $\overline{\mathrm{co}} \sigma$, which is the largest function below $\sigma$ that is lower semicontinuous in $(t, \xi)$ and sublinear in $\xi$. In Theorem 3.5, we show that the nonemptiness of $V(x, d \mid \mathbf{Q})$ is equivalent to the boundedness below of either the values of $\sigma(x, d \mid Q)$ or of the values of $\overline{\operatorname{co}} \sigma(x, d \mid Q)$. Combining these two theorems, we deduce that $\overline{\operatorname{co}} \sigma(x, d \mid Q)$, which is in terms of $\delta^{*}(\cdot \mid Q(\cdot))$, is the function $q(\cdot, \cdot)$, defined in (1.7). Therefore, a thorough answer to $\left(^{*}\right)$ is obtained. Furthermore, we show in Corollary 3.9 that not only $V(x, d \mid \mathbf{Q})$, but also $\mathrm{cl} V(x, d \mid \mathbf{Q})$ can be characterized in terms of $\overline{\operatorname{co}} \sigma(x, d \mid Q)$. In section 4 , we show how our results extend the results known in the literature for the special case when $Q(t)$ is the cone of nonnegative numbers. Specifically, a comparison with the relevant results of [6] and [7] is displayed. In section 5, we apply the results of section 3 to an optimization problem, which was the initial motivation for those results. There, Theorem 5.1 presents second-order optimality conditions in terms of the new function $\overline{\mathrm{co}} \sigma$. At the end of this section, we also present an example illustrating how the function $\overline{\mathrm{co}} \sigma$ can be evaluated.

2. Preliminary results from convex analysis. Let $T$ be a compact Hausdorff space and $\Gamma(T)$ be the set of lower semicontinuous set-valued maps $Q(\cdot)$ from $T$ to the subsets of $\mathbf{R}^{r}$ such that $Q(t) \neq \emptyset$ is closed and convex for all $t \in T$. Consider $\Lambda(T)$ the set of jointly lower semicontinuous functions $\left.\left.q: T \times \mathbf{R}^{r} \rightarrow\right]-\infty,+\infty\right]$ such that $q(t, \cdot)$ is sublinear on $\mathbf{R}^{r}$ for all $t \in T$. Set $\Sigma(T)$ to be the set of nonempty, closed, and $C$-convex subsets $\mathbf{Q}$ of $C\left(T, \mathbf{R}^{r}\right)$, where the concept of $C$-convexity is defined as

$$
\lambda(\cdot) x(\cdot)+(1-\lambda(\cdot)) y(\cdot) \in \mathbf{Q}
$$

whenever $x(\cdot), y(\cdot) \in \mathbf{Q}$, and $\lambda(\cdot) \in C(T,[0,1])$.

In [12] it is shown that to any $Q(\cdot) \in \Gamma(T)$ there corresponds a function $q(\cdot, \cdot) \in$ $\Lambda(T)$ and a set $\mathbf{Q} \in \Sigma(T)$ as follows:

$$
q(t, \xi):=\delta^{*}(\xi \mid Q(t)) \quad \text { on } T \times \mathbf{R}^{r},
$$

where $\delta^{*}$ is the support functional and

$$
\mathbf{Q}:=\left\{x(\cdot) \in C\left(T, \mathbf{R}^{r}\right) \mid x(t) \in Q(t) \forall t \in T\right\} .
$$

Furthermore, the above correspondences between the three sets are bijective and their inverses, respectively, are given by the following formulae:

$$
Q(t)=\left\{x \in \mathbf{R}^{r} \mid q(t, \xi) \geq\langle\xi, x\rangle \forall \xi \in \mathbf{R}^{r}\right\}
$$

and

$$
Q(t)=\{x(t) \mid x(\cdot) \in \mathbf{Q}\} .
$$

(Cf. [12, Theorems 4.1 and 4.2].)

Since $\mathbf{Q}$ defined by $(2.2)$ is a convex subset of $C\left(T, \mathbf{R}^{r}\right)$, then its support functional is defined by

$$
\delta^{*}(\mu \mid \mathbf{Q}):=\sup \left\{\int_{T}\langle x(t), d \mu(t)\rangle \mid x(\cdot) \in \mathbf{Q}\right\}
$$


where $\mu$ is a Radon measure on $T$ with values in $\mathbf{R}^{r}$.

The following connections between the support functionals $\delta^{*}(\cdot \mid \mathbf{Q})$ and $\delta^{*}(\cdot \mid Q(t))$, the normal cones $N(\cdot \mid \mathbf{Q})$ and $N(\cdot \mid Q(t))$, and the tangent cones $T(\cdot \mid \mathbf{Q})$ and $T(\cdot \mid Q(t))$ are derived in [16] and [12].

Let $\mu \in\left(C\left(T, \mathbf{R}^{r}\right)\right)^{*}$ be a vector-valued Radon measure on $T$ with components $\left(\mu_{1}, \ldots, \mu_{r}\right)$. Then denote

$$
|\mu|:=\left|\mu_{1}\right|+\cdots+\left|\mu_{r}\right|,
$$

where $\left|\mu_{i}\right|=\mu_{i}^{+}+\mu_{i}^{-}$, and $\mu_{i}^{+}$and $\mu_{i}^{-}$are, respectively, the positive and negative parts in the Jordan decomposition of the measure $\mu_{i}$. It is clear that $\mu$ is absolutely continuous with respect to the scalar measure $|\mu|$. We denote by $\frac{d \mu}{d|\mu|}(\cdot)$ the RadonNikodym derivative of $\mu$ with respect to $|\mu|$.

Theorem 2.1 (see [12], [16]). Let $Q(\cdot) \in \Gamma(T)$ and let $\mathbf{Q}$ be defined by (2.2). Then, for $\mu \in\left(C\left(T, \mathbf{R}^{r}\right)\right)^{*}$ and $x(\cdot) \in \mathbf{Q}$,

(i) $\delta^{*}(\mu \mid \mathbf{Q})=\int_{T} \delta^{*}\left(\frac{d \mu}{d|\mu|}(t) \mid Q(t)\right) d|\mu|(t)$,

(ii) $\mu \in N(x(\cdot) \mid \mathbf{Q})$ iff $\frac{d \mu}{d|\mu|}(t) \in N(x(t) \mid Q(t)) \quad|\mu|$-a.e.,

(iii) for $u(\cdot) \in C\left(T, \mathbf{R}^{r}\right)$,

$$
u(\cdot) \in T(x(\cdot) \mid \mathbf{Q}) \quad \text { iff } \quad u(t) \in T(x(t) \mid Q(t)) \quad \forall t \in T .
$$

Now consider the following subsets of $\Gamma(T), \Lambda(T)$, and $\Sigma(T)$ :

$$
\begin{aligned}
& \Gamma_{0}(T):=\{Q(\cdot) \in \Gamma(T) \mid \operatorname{int} Q(t) \neq \emptyset \forall t \in T\}, \\
& \Lambda_{0}(T):=\{q \in \Lambda(T) \mid q(t, \xi)+q(t,-\xi)>0 \forall \xi \neq 0, \forall t \in T\}, \\
& \Sigma_{0}(T):=\{\mathbf{Q} \in \Sigma(T) \mid \operatorname{int} \mathbf{Q} \neq \emptyset\} .
\end{aligned}
$$

The following result was developed in [12]. It states that the three subsets defined above are also equivalent. Furthermore, the connections between the interiors of the sets $\mathbf{Q}$ and $Q(t)$ and the set

$$
\left\{x \in \mathbf{R}^{r} \mid q(t, \xi)>\langle\xi, x\rangle \forall \xi \neq 0\right\}
$$

are presented.

TheOREm 2.2 (see [12, Theorems 4.1 and 4.2]). The sets $\Gamma_{0}(T), \Lambda_{0}(T)$, and $\Sigma_{0}(T)$ are equivalent via the maps defined in (2.1) and (2.2). Furthermore, for $Q(\cdot) \in$ $\Gamma_{0}(T)$, we have

$$
\operatorname{int} Q(t)=\left\{x \in \mathbf{R}^{r} \mid q(t, \xi)>\langle\xi, x\rangle \quad \forall \xi \neq 0\right\} \quad \forall t \in T
$$

and

$$
\operatorname{int} \mathbf{Q}=\left\{x(\cdot) \in C\left(T, \mathbf{R}^{r}\right) \mid x(t) \in \operatorname{int} Q(t) \forall t \in T\right\} \neq \emptyset,
$$

where $q$ and $\mathbf{Q}$ are defined in (2.1) and (2.2), respectively.

Using the results of Theorem 2.2, the relationship between nonempty open $C$ convex subsets of $C\left(T, \mathbf{R}^{r}\right)$, lower semicontinuous set-valued maps with nonempty open and convex images, and functionals in $\Lambda_{0}(T)$ can also be established. Let

$$
\begin{aligned}
\boldsymbol{\Gamma}_{0}(T):=\left\{Q(\cdot): T \rightarrow 2^{\mathbf{R}^{r}} \mid Q(\cdot) \text { is lsc and } Q(t) \neq \emptyset \text { is open and convex } \forall t \in T\right\}, \\
\boldsymbol{\Sigma}_{0}(T):=\left\{\mathbf{Q} \subset C\left(T, \mathbf{R}^{r}\right) \mid \mathbf{Q} \neq \emptyset \text { is open and } C \text {-convex }\right\} .
\end{aligned}
$$


Corollary 2.3 (see [12, Corollary 4.4]). The set $\boldsymbol{\Gamma}_{0}(T)$ is equivalent to $\Lambda_{0}(T)$ and $\boldsymbol{\Sigma}_{0}(T)$ via the correspondences defined by (2.1) and (2.2), respectively.

REMARK 2.1. Let $X$ be a Banach space and let $\mathbf{Q} \subset X$ be a convex set with nonempty interior and $x \in \mathbf{Q}$. Then a vector $d \in X$ is called a first-order admissible variation of $\mathbf{Q}$ at $x$ if there exists $\bar{\varepsilon}>0$ such that

$$
x+\varepsilon(d+w) \in \mathbf{Q} \quad \text { for all } \quad 0<\varepsilon<\bar{\varepsilon},\|w\|<\bar{\varepsilon}, w \in X .
$$

The set of first-order admissible variations is the Dubovitskii-Milyutin cone defined in [3]. A thorough investigation of this cone is important in obtaining first-order necessary conditions concerning inequality constraints (see [4]). It is also known (cf. $[4$, Theorem 8.2]) that this cone is given by

$$
\{\lambda(u-x) \mid u \in \operatorname{int} \mathbf{Q}, \lambda>0\} .
$$

It is easy to see that the closure of this set is equal to the cone $T(x \mid \mathbf{Q})$. Thus the set of first-order admissible variations can also be represented as the interior of $T(x \mid \mathbf{Q})$.

Consider the case $X=C\left(T, \mathbf{R}^{r}\right)$ and $\mathbf{Q}$ given by $(2.2)$ for $Q(\cdot)$ in $\Gamma(T)$. Thus, by combining Theorems 2.1 and 2.2 , we obtain that $d(\cdot)$ is an admissible first-order variation with respect to the set $\mathbf{Q}$ at $x(\cdot) \in \mathbf{Q}$ if and only if

$$
d(t) \in \operatorname{int} T(x(t) \mid Q(t)) \quad \text { for } \quad t \in T .
$$

This represents a characterization of the set of first-order variations of $\mathbf{Q}$ in terms of $Q(\cdot)$.

3. Second-order admissible variations. Let $X$ be a Banach space and let $\mathbf{Q} \subset X$ be a closed convex set. In addition, let $x \in \mathbf{Q}$ and $d \in X$. As defined in section 1, a vector $v \in X$ is called a second-order admissible variation of $\mathbf{Q}$ at $x$ in the direction $d$ if there exists $\bar{\varepsilon}>0$ such that

$$
x+\varepsilon d+\varepsilon^{2}(v+w) \in \mathbf{Q} \quad \text { for all } \quad 0<\varepsilon<\bar{\varepsilon},\|w\|<\bar{\varepsilon}, w \in X .
$$

In the sequel, set $X=C\left(T, \mathbf{R}^{r}\right), T=(T, \rho)$ to be a compact metric space, and set $Q: T \rightarrow 2^{\mathbf{R}^{r}}$ to be a lower semicontinuous set-valued function whose images are closed and convex with nonempty interior. Define the set $\mathbf{Q} \subset C\left(T, \mathbf{R}^{r}\right)$ by

$$
\mathbf{Q}=\left\{x(\cdot) \in C\left(T, \mathbf{R}^{r}\right) \mid x(t) \in Q(t)\right\} .
$$

Then int $\mathbf{Q} \neq \emptyset$ by Theorem 2.2 .

The main results of this paper are given in this section. We focus on characterizing the set $V(x, d \mid \mathbf{Q})$ in terms of the images $Q(t)$ and their support functionals. As we shall see this is obtained through the function $\sigma$.

Let $x \in \mathbf{Q}$ and $d \in T(x \mid \mathbf{Q})$ be arbitrarily fixed continuous functions. In order to describe $V(x, d \mid \mathbf{Q})$, we have to introduce now the following notation.

Let

$$
\mathcal{C}(x, d \mid Q):=\left\{(t, \xi) \in T \times \mathbf{R}^{r} \mid \xi \in N(x(t) \mid Q(t)),\langle\xi, d(t)\rangle=0\right\}
$$

and

$$
\mathcal{C}(x, d \mid Q)(t):=\left\{\xi \in \mathbf{R}^{r} \mid(t, \xi) \in \mathcal{C}(x, d \mid Q)\right\} .
$$


Lemma 3.1. The set-valued map $\mathcal{C}(x, d \mid Q)(\cdot)$ is nonempty closed convex cone valued; furthermore, $\mathcal{C}(x, d \mid Q)$ is a closed set.

Proof. We have

$$
\mathcal{C}(x, d \mid Q)(t)=N(x(t) \mid Q(t)) \cap\{d(t)\}^{\perp} .
$$

Hence $\mathcal{C}(x, d \mid Q)(t)$ is a nonempty closed convex cone. It remains to prove only the closedness of $\mathcal{C}(x, d \mid Q)$. Let $t_{n} \rightarrow t_{0}$ and $\xi_{n} \in \mathcal{C}(x, d \mid Q)\left(t_{n}\right)$ with $\xi_{n} \rightarrow \xi_{0}$ be arbitrary sequences. Then, by (3.2),

$$
\left\langle\xi_{n}, x\left(t_{n}\right)\right\rangle=\delta^{*}\left(\xi_{n} \mid Q\left(t_{n}\right)\right)\left\langle\xi_{n}, d\left(t_{n}\right)\right\rangle=0 .
$$

Using the lower semicontinuity of $\delta^{*}(\cdot \mid Q(\cdot))$ (see [12, Lemma 3.3]) and upon taking the liminf, we obtain

$$
\left\langle\xi_{0}, x\left(t_{0}\right)\right\rangle \geq \delta^{*}\left(\xi_{0} \mid Q\left(t_{0}\right)\right) \text { and }\left\langle\xi_{0}, d\left(t_{0}\right)\right\rangle=0 .
$$

The first inequality can only be equality since $x\left(t_{0}\right) \in Q\left(t_{0}\right)$ and $\left\langle\xi_{0}, x\left(t_{0}\right)\right\rangle \leq \delta^{*}\left(\xi_{0} \mid Q\left(t_{0}\right)\right)$ is always true. Thus $\xi_{0} \in \mathcal{C}(x, d \mid Q)\left(t_{0}\right)$, from which the closedness of $\mathcal{C}(x, d \mid Q)$ follows.

Let

$$
\mathcal{S}(x, d \mid Q):=\left\{(t, \xi) \in T \times \mathbf{R}^{r} \mid \delta^{*}(\xi \mid Q(t))>\langle\xi, x(t)\rangle,\langle\xi, d(t)\rangle>0\right\},
$$

and denote by $\mathcal{S}^{\prime}(x, d \mid Q)$ the set of accumulation points of $\mathcal{S}(x, d \mid Q)$; i.e.,

$$
\mathcal{S}^{\prime}(x, d \mid Q)=\left\{(t, \xi) \mid \exists t_{n} \rightarrow t, \exists \xi_{n} \rightarrow \xi \text { with }\left(t_{n}, \xi_{n}\right) \in \mathcal{S}(x, d \mid Q) \forall n\right\} .
$$

Then, obviously, $\mathcal{S}(x, d \mid Q)$ is an open subset of $T \times \mathbf{R}^{r}$. Define

$$
\sigma(x, d \mid Q)(t, \xi):=\left\{\begin{array}{cc}
\liminf _{(s, \zeta) \rightarrow(t, \xi)} E(x, d \mid Q)(s, \zeta), \text { if }(t, \xi) \in \mathcal{C}(x, d \mid Q) \cap \mathcal{S}^{\prime}(x, d \mid Q), \\
(s, \zeta) \in \mathcal{S}(x, d \mid Q) & \text { if }(t, \xi) \in \mathcal{C}(x, d \mid Q) \backslash \mathcal{S}^{\prime}(x, d \mid Q), \\
0 & \text { otherwise, }
\end{array}\right.
$$

where

$$
E(x, d \mid Q)(t, \xi):=\frac{[\langle\xi, d(t)\rangle]^{2}}{4\left[\langle\xi, x(t)\rangle-\delta^{*}(\xi \mid Q(t))\right]} .
$$

REMARK 3.1. First, note that the ratio $E(x, d \mid Q)(t, \xi)$ is positively homogeneous in $\xi$; therefore, the liminf in the definition of the function $\sigma(x, d \mid Q)$ could be restricted to those $\zeta$ with $|\zeta|=|\xi|$ if $\xi \neq 0$. Furthermore, $\mathcal{C}(x, d \mid Q)(t)$ and $\mathcal{S}(x, d \mid Q)(t)$, which is defined similarly to $\mathcal{C}(x, d \mid Q)(t)$, are cones; thus it follows that $\sigma(x, d \mid Q)(t, \cdot)$ is a positively homogeneous function. As we shall see in Lemma 3.3 below, the function $\sigma(x, d \mid Q)(\cdot, \cdot)$ is in fact lower semicontinuous on $T \times\left(\mathbf{R}^{r} \backslash\{0\}\right)$.

Define the convex regularization of $\sigma(x, d \mid Q)(t, \cdot)$ by

$$
\begin{aligned}
\operatorname{co} \sigma(x, d \mid Q)(t, \xi):=\inf \left\{\sum_{i=1}^{n} \lambda_{i} \sigma(x, d \mid Q)\left(t, \xi_{i}\right) \mid\right. \\
\left.\xi=\sum_{i=1}^{n} \lambda_{i} \xi_{i}, \xi_{i} \in \mathbf{R}^{r}, \lambda_{i} \in[0,1], \sum_{i=1}^{n} \lambda_{i}=1\right\} .
\end{aligned}
$$


Hence $\operatorname{co} \sigma$ is the largest convex function in $\xi$ such that

$$
\operatorname{co} \sigma(x, d \mid Q)(t, \xi) \leq \sigma(x, d \mid Q)(t, \xi) \quad \text { for } \quad t \in T, \xi \in \mathbf{R}^{r} .
$$

Consequently, $\operatorname{co} \sigma(x, d \mid Q)(t, \cdot)$ is sublinear for each fixed $t \in T$.

Now consider the lower semicontinuous regularization in $(t, \xi)$ of $\operatorname{co} \sigma$. It is denoted by $\overline{\mathrm{co}} \sigma$ and is defined as

$$
\overline{\operatorname{co}} \sigma(x, d \mid Q)(t, \xi):=\liminf _{(\tau, \zeta) \rightarrow(t, \xi)} \operatorname{co} \sigma(x, d \mid Q)(\tau, \zeta) .
$$

Clearly, $\overline{\mathrm{co}} \sigma$ is the largest lower semicontinuous function in $(t, \xi)$ that is bounded above by $\operatorname{co} \sigma$. The result is that $\overline{\operatorname{co}} \sigma(x, d \mid Q)(t, \cdot)$ is also sublinear. Therefore, $\overline{\operatorname{co}} \sigma$ is in $\Lambda(T)$.

The aim of this section is twofold:

(i) to establish a characterization of the set $V(x, d \mid \mathbf{Q})$ in terms of the convex and jointly lower semicontinuous function $\overline{\operatorname{co}} \sigma(x, d \mid Q)$ (see Theorem 3.2);

(ii) to provide a full description of the nonemptiness of $V(x, d \mid \mathbf{Q})$ in terms of the function $\sigma(x, d \mid Q)$ or $\overline{\operatorname{co}} \sigma(x, d \mid Q)$ (see Theorem 3.5).

The combination of these results confirms that $\overline{\mathrm{co}} \sigma$ is the function $q$ in (1.7) corresponding to the set-valued map $V(x, d \mid \mathbf{Q})(\cdot)$. Another consequence is that $\overline{\mathrm{co}} \sigma$ is also the function $q$ in $(2.1)$ corresponding to the set-valued map $\bar{V}(x, d \mid \mathbf{Q})(\cdot)$, where

$$
\bar{V}(x, d \mid \mathbf{Q})(t):=\left\{v(t) \in \mathbf{R}^{r} \mid v(\cdot) \in \operatorname{cl} V(x, d \mid \mathbf{Q})\right\}
$$

(see Corollary 3.9).

Theorem 3.2. A function $v: T \rightarrow \mathbf{R}^{r}$ belongs to $V(x, d \mid \mathbf{Q})$ if and only if

$$
\langle\xi, v(t)\rangle<\overline{\operatorname{co}} \sigma(x, d \mid Q)(t, \xi) \quad \text { for all } t \in T, \xi \in\left(\mathbf{R}^{r} \backslash\{0\}\right) .
$$

The proof is based on the following two results. The first is a modification of the key lemma from [11].

Lemma 3.3. Let $\mathcal{T}$ be a compact metric space and let $a: \mathcal{T} \rightarrow[-\infty, \infty[$ and $b, c: \mathcal{T} \rightarrow \mathbf{R}$ be upper semicontinuous functions. Define $\sigma_{a, b}: \mathcal{T} \rightarrow[-\infty, \infty]$ by

$$
\sigma_{a, b}(u):=\left\{\begin{array}{cl}
\liminf _{\substack{\tau \rightarrow u \\
a(\tau)<0, b(\tau)>0}} \frac{b^{2}(\tau)}{4 a(\tau)} & \text { if } u \in \mathcal{T}_{a=0, b=0} \cap \partial\left(\mathcal{T}_{a<0, b>0}\right), \\
0 & \text { if } u \in \mathcal{T}_{a=0, b=0} \backslash \partial\left(\mathcal{T}_{a<0, b>0}\right), \\
+\infty & \text { otherwise. }
\end{array}\right.
$$

Then $\sigma_{a, b}$ is a lower semicontinuous function and the set of points where $\sigma_{a, b}<0$ is nowhere dense in $\mathcal{T}$. Furthermore, the following statements are equivalent to each other

(i)

$$
\begin{array}{ll}
a(u) \leq 0 & \text { for all } u \in \mathcal{T}, \\
b(u) \leq 0 & \text { for all } u \in \mathcal{T}_{a=0}, \\
c(u)<\sigma_{a, b}(u) & \text { for all } u \in \mathcal{T} .
\end{array}
$$

(ii) There exists $\delta>0$ such that, for all $u_{0} \in \mathcal{T}$, for all sequences $u_{n} \in \mathcal{T}$ with $u_{n} \rightarrow u_{0}$ and $\varepsilon_{n}>0$ with $\varepsilon_{n} \rightarrow 0$,

$$
a\left(u_{n}\right)+\varepsilon_{n} b\left(u_{n}\right)+\varepsilon_{n}^{2}\left(c\left(u_{0}\right)+\delta\right) \leq 0
$$

holds for sufficiently large values of $n \in \mathbf{N}$. 
If $c$ is also continuous, then the following statement is also equivalent to (i) and (ii).

(iii) There exists $\bar{\varepsilon}>0$ such that, for all $u \in \mathcal{T}$ and for all $\varepsilon<\bar{\varepsilon}$,

$$
a(u)+\varepsilon b(u)+\varepsilon^{2}(c(u)+\bar{\varepsilon}) \leq 0 .
$$

REMARK 3.2. The novelty in the formulation of this lemma versus the key lemma in [11] resides in the fact that the functions $a$ and $\sigma_{a, b}$ may take the value $-\infty$ and $+\infty$, respectively, and in the equivalence of the third condition (iii) with each of (i) and (ii).

REMARK 3.3. The function $\sigma_{a, b}$ and the function $\mathbf{E}=\mathbf{E}_{x, d}$ introduced in Theorem 1.2 satisfy the following identity (if $x, d$ are as in Theorem 1.2):

$$
\mathbf{E}_{x, d}=-\sigma_{-x,-d} .
$$

This relation can be checked immediately. Therefore, the use of the function $\mathbf{E}$ is also possible if $a, b$ are continuous functions.

Proof. The proof of the lower semicontinuity of $\sigma_{a, b}$ and (i) $\Longleftrightarrow(i i)$ is analogous to that of the key lemma in [11].

(ii) $\Rightarrow$ (iii). Assume that (iii) is not true. Then, for all $\bar{\varepsilon}=1 / n$, there exists $u_{n} \in \mathcal{T}, \varepsilon_{n}<1 / n$ such that

$$
a\left(u_{n}\right)+\varepsilon_{n} b\left(u_{n}\right)+\varepsilon_{n}^{2}\left(c\left(u_{n}\right)+\frac{1}{n}\right)>0 .
$$

We may assume that $u_{n} \rightarrow u_{0}$, since $\mathcal{T}$ is compact. By the upper semicontinuity of $c, c\left(u_{n}\right)+1 / n \leq c\left(u_{0}\right)+\delta$ for large $n$. Thus

$$
a\left(u_{n}\right)+\varepsilon_{n} b\left(u_{n}\right)+\varepsilon_{n}^{2}\left(c\left(u_{0}\right)+\delta\right)>0
$$

for large $n$, which contradicts (ii). (iii),

(iii) $\Rightarrow\left(\right.$ ii). Let $\delta:=\bar{\varepsilon} / 2$ and $u_{n} \rightarrow u_{0}, \varepsilon_{n} \rightarrow 0$ be arbitrary sequences. Then, by

$$
a\left(u_{n}\right)+\varepsilon_{n} b\left(u_{n}\right)+\varepsilon_{n}^{2}\left(c\left(u_{n}\right)+\bar{\varepsilon}\right) \leq 0
$$

for $\varepsilon_{n}<\bar{\varepsilon}$, that is, for large values of $n$. Since $c$ is lower semicontinuous, $c\left(u_{n}\right)>$ $c\left(u_{0}\right)-\delta$ if $n$ is large enough. Thus $c\left(u_{n}\right)+\bar{\varepsilon}>c\left(u_{0}\right)+\delta$ and therefore

$$
a\left(u_{n}\right)+\varepsilon_{n} b\left(u_{n}\right)+\varepsilon_{n}^{2}\left(c\left(u_{0}\right)+\delta\right) \leq 0
$$

for sufficiently large $n$.

The following result is the second key to prove Theorem 3.2. It is a characterization of $V(x, d \mid \mathbf{Q})$ in terms of $\sigma$. Hence it is a weaker result than Theorem 3.2. if

Lemma 3.4. A continuous function $v: T \rightarrow \mathbf{R}^{r}$ belongs to $V(x, d \mid \mathbf{Q})$ if and only

$$
\langle\xi, v(t)\rangle<\sigma(x, d \mid Q)(t, \xi) \quad \text { for all } \quad t \in T, \xi \in \mathbf{R}^{r} \backslash\{0\} .
$$

Proof. A function $v: T \rightarrow \mathbf{R}^{r}$ belongs to $V(x, d \mid \mathbf{Q})$ if and only if there exists $\bar{\varepsilon}>0$ such that

$$
x(t)+\varepsilon d(t)+\varepsilon^{2}(v(t)+w(t)) \in Q(t)
$$


for all $t \in T, \varepsilon \leq \bar{\varepsilon}$ and continuous functions $w: T \rightarrow \mathbf{R}^{r}$ with $\|w\| \leq \bar{\varepsilon}$. The set $Q(t)$ is closed and convex; therefore, $p \in Q(t)$ holds if and only if $\langle\xi, p\rangle \leq \delta^{*}(\xi \mid Q(t))$ for all $\xi \in \mathbf{R}^{r}$ with $|\xi|=1$. Applying this argument, we obtain that $v \in V(x, d \mid \mathbf{Q})$ if and only if

$$
\langle\xi, x(t)\rangle+\varepsilon\langle\xi, d(t)\rangle+\varepsilon^{2}(\langle\xi, v(t)\rangle+\bar{\varepsilon}) \leq \delta^{*}(\xi, Q(t))
$$

for all $t \in T, \varepsilon \leq \bar{\varepsilon}$ and $\xi$ with $|\xi|=1$.

Take

$$
\begin{aligned}
\mathcal{T} & :=T \times\left\{\xi \in \mathbf{R}^{r}:|\xi|=1\right\}, \\
a(t, \xi) & :=\langle\xi, x(t)\rangle-\delta^{*}(\xi \mid Q(t)), \\
b(t, \xi) & :=\langle\xi, d(t)\rangle, \\
c(t, \xi) & :=\langle\xi, v(t)\rangle .
\end{aligned}
$$

Then $\mathcal{T}$ is a compact metric space, and the functions $a, b, c$ satisfy the conditions of Lemma 3.3. One can also observe that

$$
\sigma(x, d \mid Q)(t, \xi)=\sigma_{a, b}(t, \xi) \quad \text { for } \quad(t, \xi) \in \mathcal{T} .
$$

Therefore, by the equivalence of the statements (i) and (iii) of Lemma 3.3, $v$ satisfies (3.9) on the domain indicated if and only if

$$
\begin{gathered}
\quad\langle\xi, x(t)\rangle-\delta^{*}(\xi \mid Q(t)) \leq 0 \quad \text { for } \quad(t, \xi) \in \mathcal{T}, \\
\langle\xi, d(t)\rangle \leq 0 \quad \text { for } \quad(t, \xi) \in \mathcal{T} \text { such that }\langle\xi, x(t)\rangle=\delta^{*}(\xi \mid Q(t)),
\end{gathered}
$$

and

$$
\langle\xi, v(t)\rangle<\sigma(x, d \mid Q)(t, \xi) \quad \text { for } \quad(t, \xi) \in \mathcal{T} .
$$

The first inequality immediately follows from $x(t) \in Q(t)$. The relation $\langle\xi, x(t)\rangle=$ $\delta^{*}(\xi \mid Q(t))$ is equivalent to $\xi \in N(x(t) \mid Q(t))$; therefore, the second inequality is equivalent to $d(t) \in T(x(t) \mid Q(t))$ which by Theorem 2.1 is the same as $d \in T(x \mid \mathbf{Q})$. Thus $v \in V(x, d \mid \mathbf{Q})$ if and only if (3.11) holds. Using the observation on the positive homogeneity in Remark 3.1, we get the statement of this lemma.

Proof of Theorem 3.2 (Sufficiency). If $v$ satisfies (3.8), then (3.5) and (3.6) yield that $v$ also satisfies the condition of Lemma 3.4; therefore, $v \in V(x, d \mid \mathbf{Q})$.

Necessity. Assume that $v \in V(x, d \mid \mathbf{Q})$. Since the set $V(x, d \mid \mathbf{Q})$ is open, then there exists $\delta>0$ such that $v+\delta \mathbf{B} \subset V(x, d \mid \mathbf{Q})$. Thus, by Lemma 3.4,

$$
\langle\xi, v(t)\rangle+\delta|\xi|<\sigma(x, d \mid Q)(t, \xi) \quad \text { for all } \quad t \in T, \xi \in\left(\mathbf{R}^{r} \backslash\{0\}\right) .
$$

By defining $\sigma(x, d \mid Q)(t, 0):=0$, the domain of this inequality can be extended to the set $T \times \mathbf{R}^{r}$.

Let $\xi_{1}, \ldots, \xi_{n} \in \mathbf{R}^{r}$ and $\lambda_{1}, \ldots, \lambda_{n} \in[0,1]$ with $\lambda_{1}+\cdots+\lambda_{n}=1$. Then it follows from (3.12) that

$$
\left\langle\sum_{i=1}^{n} \lambda_{i} \xi_{i}, v(t)\right\rangle+\delta\left|\sum_{i=1}^{n} \lambda_{i} \xi_{i}\right| \leq \sum_{i=1}^{n} \lambda_{i}\left[\left\langle\xi_{i}, v(t)\right\rangle+\delta\left|\xi_{i}\right|\right] \leq \sum_{i=1}^{n} \lambda_{i} \sigma(x, d \mid Q)\left(t, \xi_{i}\right) .
$$


Therefore,

$$
\begin{aligned}
& \langle\xi, v(t)\rangle+\delta|\xi| \leq \operatorname{co} \sigma(x, d \mid Q)(t, \xi) \\
& \quad=\inf \left\{\sum_{i=1}^{n} \lambda_{i} \sigma(x, d \mid Q)\left(t, \xi_{i}\right) \mid \xi=\sum_{i=1}^{n} \lambda_{i} \xi_{i}, \xi_{i} \in \mathbf{R}^{r}, \lambda_{i} \in[0,1], \sum_{i=1}^{n} \lambda_{i}=1\right\} .
\end{aligned}
$$

Now fix $\left(t_{0}, \xi_{0}\right)$ and take the liminf with respect to $(t, \xi) \rightarrow\left(t_{0}, \xi_{0}\right)$ in the above inequality. We get

$$
\left\langle\xi_{0}, v\left(t_{0}\right)\right\rangle+\delta\left|\xi_{0}\right| \leq \liminf _{(t, \xi) \rightarrow\left(t_{0}, \xi_{0}\right)} \operatorname{co} \sigma(x, d \mid Q)(t, \xi)=\overline{\operatorname{co}} \sigma(x, d \mid Q)\left(t_{0}, \xi_{0}\right) .
$$

Since $\left(t_{0}, \xi_{0}\right)$ is arbitrary, (3.8) follows at once.

The statement below is a characterization of the nonemptiness of $V(x, d \mid \mathbf{Q})$.

THEOREM 3.5. The following three statements are equivalent:

(i) $V(x, d \mid \mathbf{Q}) \neq \emptyset$,

(ii) $\overline{\operatorname{co}} \sigma(x, d \mid Q)(t, \xi)>-\infty \quad$ for all $(t, \xi) \in \mathcal{T}$,

(iii) $\sigma(x, d \mid Q)(t, \xi)>-\infty \quad$ for all $(t, \xi) \in \mathcal{T}$.

Proof. (i) $\Longrightarrow$ (ii). If $v \in V(x, d \mid \mathbf{Q})$, then by Theorem 3.2,

$$
\langle\xi, v(t)\rangle<\overline{\operatorname{co}} \sigma(x, d \mid Q)(t, \xi) \quad \text { for all } \quad(t, \xi) \in \mathcal{T} .
$$

Hence $\overline{\mathrm{co}} \sigma(x, d \mid Q)(t, \xi)>-\infty$.

The implication (ii) $\Longrightarrow$ (iii) is obvious. The proof of (iii) $\Longrightarrow($ i) is split into three steps.

Step 1. We prove the following lemma.

Lemma 3.6. If (iii) holds, then there exists $K>0$ such that

$$
-K|\xi| \leq E(x, d \mid Q)(t, \xi)
$$

if $(t, \xi) \in \mathcal{S}(x, d \mid Q)$. (Here $E(x, d \mid Q)(\cdot, \cdot)$ is defined in (3.4).)

Proof. By contradiction assume that for all $n \in \mathbf{N}$ there exists $\left(t_{n}, \xi_{n}\right) \in \mathcal{S}(x, d \mid Q)$ with $\left|\xi_{n}\right|=1$ so that

$$
E(x, d \mid Q)\left(t_{n}, \xi_{n}\right)<-n .
$$

We can assume that $t_{n} \rightarrow t_{0}$ and $\xi_{n} \rightarrow \xi_{0}$. Observe that the function $E(x, d \mid Q)(\cdot, \cdot)$ is lower semicontinuous; therefore, we obtain

$$
-\infty=\liminf _{n \rightarrow \infty} E(x, d \mid Q)\left(t_{n}, \xi_{n}\right) \geq E(x, d \mid Q)\left(t_{0}, \xi_{0}\right) .
$$

That is, $E(x, d \mid Q)\left(t_{0}, \xi_{0}\right)=-\infty$. Thus $\left(t_{0}, \xi_{0}\right) \notin \mathcal{S}(x, d \mid Q)$. The sequence $\left(t_{n}, \xi_{n}\right)$ being in this set, $\left(t_{0}, \xi_{0}\right)$ must belong to the boundary of $\mathcal{S}(x, d \mid Q)$. This means that at least one of the equalities

$$
\delta^{*}\left(\xi_{0} \mid Q\left(t_{0}\right)\right)=\left\langle\xi_{0}, x\left(t_{0}\right)\right\rangle, \quad\left\langle\xi_{0}, d\left(t_{0}\right)\right\rangle=0
$$

is valid. The relations

$$
\delta^{*}\left(\xi_{0} \mid Q\left(t_{0}\right)\right)=\left\langle\xi_{0}, x\left(t_{0}\right)\right\rangle \text { and }\left\langle\xi_{0}, d\left(t_{0}\right)\right\rangle>0
$$

are not possible because $d\left(t_{0}\right)$ is tangent to $Q\left(t_{0}\right)$ at $x\left(t_{0}\right)$. If

$$
\delta^{*}\left(\xi_{0} \mid Q\left(t_{0}\right)\right)>\left\langle\xi_{0}, x\left(t_{0}\right)\right\rangle \text { and }\left\langle\xi_{0}, d\left(t_{0}\right)\right\rangle=0,
$$


then $E(x, d \mid Q)\left(t_{0}, \xi_{0}\right)=0$, which is a contradiction. The last case is when

$$
\delta^{*}\left(\xi_{0} \mid Q\left(t_{0}\right)\right)=\left\langle\xi_{0}, x\left(t_{0}\right)\right\rangle \text { and }\left\langle\xi_{0}, d\left(t_{0}\right)\right\rangle=0 .
$$

Then $\left(t_{0}, \xi_{0}\right) \in \mathcal{C}(x, d \mid Q)$. Therefore,

$$
-\infty=\liminf _{n \rightarrow \infty} E(x, d \mid Q)\left(t_{n}, \xi_{n}\right) \geq \sigma(x, d \mid Q)\left(t_{0}, \xi_{0}\right)>-\infty,
$$

which means a contradiction to (iii).

Step 2. We express the characterization obtained in Lemma 3.4 in a different form.

Lemma 3.7. A function $v: T \rightarrow \mathbf{R}^{r}$ belongs to $V(x, d \mid \mathbf{Q})$ if and only if there exists a positive number $\bar{\varepsilon}>0$ such that

$$
\langle\xi, v(t)\rangle \leq\left\{\begin{array}{r}
-\bar{\varepsilon}|\xi|+E(x, d \mid Q)(t, \xi) \\
\text { if } \bar{\varepsilon}\langle\xi, d(t)\rangle>2\left[\delta^{*}(\xi \mid Q(t))-\langle\xi, x(t)\rangle\right], \\
-\bar{\varepsilon}|\xi|+\frac{\delta^{*}(\xi \mid Q(t))-\langle\xi, x(t)\rangle-\bar{\varepsilon}\langle\xi, d(t)\rangle}{\bar{\varepsilon}^{2}} \\
\text { if } \bar{\varepsilon}\langle\xi, d(t)\rangle \leq 2\left[\delta^{*}(\xi \mid Q(t))-\langle\xi, x(t)\rangle\right]
\end{array}\right.
$$

for all $t \in T, \xi \in\left(\mathbf{R}^{r} \backslash\{0\}\right)$.

Proof. As we have already proved in the proof of Lemma 3.4, $v \in V(x, d \mid \mathbf{Q})$ holds if and only if there exists $\bar{\varepsilon}>0$ such that (3.9) holds for all $t \in T, \varepsilon \leq \bar{\varepsilon}$, and $\xi \in \mathbf{R}^{r}$ with $|\xi|=1$. In other words, $v \in V(x, d \mid \mathbf{Q})$ if and only if there exists $\bar{\varepsilon}>0$ such that

$$
\langle\xi, v(t)\rangle \leq-\bar{\varepsilon}|\xi|+\inf _{0<\varepsilon \leq \bar{\varepsilon}} \frac{\delta^{*}(\xi \mid Q(t))-\langle\xi, x(t)\rangle-\varepsilon\langle\xi, d(t)\rangle}{\varepsilon^{2}}
$$

for all $t \in T$ and for all $\xi \neq 0$. Computing the infimum on the right-hand side, the result follows immediately.

Step 3. The following lemma completes the proof of Theorem 3.5.

Lemma 3.8. If (iii) holds, then for any $w \in \operatorname{int} \mathbf{Q}$ there exists $\bar{\varepsilon}$ so that

$$
v=\frac{w-x-\bar{\varepsilon} d}{\bar{\varepsilon}^{2}} \in V(x, d \mid \mathbf{Q}) .
$$

Proof. Let $w$ be in the interior of $\mathbf{Q}$. Then there exists $\delta>0$ such that $w+\delta \mathbf{B} \subset \mathbf{Q}$ (where $\mathbf{B}$ stands for the unit ball of the space $C\left(T, \mathbf{R}^{r}\right)$ ). Hence

$$
\langle\xi, w(t)\rangle+\delta|\xi| \leq \delta^{*}(\xi \mid Q(t)) \quad \text { for all } \quad t \in T, \xi \in \mathbf{R}^{r} .
$$

Let $K$ be the constant whose existence was stated in Lemma 3.6. Choose $\bar{\varepsilon}>0$ so that $\bar{\varepsilon}^{2}(\bar{\varepsilon}+K) \leq \delta$. Thus we have

$$
\langle\xi, w(t)\rangle+\bar{\varepsilon}^{2}(\bar{\varepsilon}+K)|\xi| \leq \delta^{*}(\xi \mid Q(t)) \quad \text { for all } t \in T, \xi \in \mathbf{R}^{r} .
$$

To complete the proof of this lemma, we are going to show that $v$ satisfies the necessary and sufficient condition of Lemma 3.7 with this $\bar{\varepsilon}$.

Substituting $v=(w-x-\bar{\varepsilon} d) / \bar{\varepsilon}^{2}$ into this condition, it remains to prove that

$$
\langle\xi, w(t)\rangle \leq\left\{\begin{array}{rr}
-\bar{\varepsilon}^{3}|\xi|+\bar{\varepsilon}^{2} E(x, d \mid Q)(t, \xi)+\langle\xi, x(t)\rangle+\bar{\varepsilon}\langle\xi, d(t)\rangle \\
-\bar{\varepsilon}^{3}|\xi|+\delta^{*}(\xi \mid Q(t)) & \text { if } \bar{\varepsilon}\langle\xi, d(t)\rangle>2\left[\delta^{*}(\xi \mid Q(t))-\langle\xi, x(t)\rangle\right], \\
& \text { if } \bar{\varepsilon}\langle\xi, d(t)\rangle \leq 2\left[\delta^{*}(\xi \mid Q(t))-\langle\xi, x(t)\rangle\right]
\end{array}\right.
$$


for all $t \in T, \xi \in\left(\mathbf{R}^{r} \backslash\{0\}\right)$.

By the choice of $\bar{\varepsilon}$,

$$
\langle\xi, w(t)\rangle+\bar{\varepsilon}^{3}|\xi| \leq \delta^{*}(\xi \mid Q(t)) \quad \text { for all } \quad t \in T, \xi \in \mathbf{R}^{r} .
$$

Therefore, it remains to show that the first inequality holds on the domain indicated.

If $(t, \xi)$ belongs to that domain, then

$$
\bar{\varepsilon}\langle\xi, d(t)\rangle>2\left[\delta^{*}(\xi \mid Q(t))-\langle\xi, x(t)\rangle\right] \geq \delta^{*}(\xi \mid Q(t))-\langle\xi, x(t)\rangle .
$$

The right-hand side of this inequality cannot be zero, otherwise $\langle\xi, d(t)\rangle=0$, which gives a contradiction. Thus $(t, \xi) \in \mathcal{S}(x, d \mid Q)$ holds too. Therefore, by Lemma 3.6, (3.13) is valid. Combining these inequalities, we obtain

$$
\begin{aligned}
\langle\xi, w(t)\rangle & \leq \delta^{*}(\xi \mid Q(t))-\bar{\varepsilon}^{2}(\bar{\varepsilon}+K)|\xi| \\
& <\bar{\varepsilon}\langle\xi, d(t)\rangle+\langle\xi, x(t)\rangle-\bar{\varepsilon}^{3}|\xi|-\bar{\varepsilon}^{2} K|\xi| \\
& \leq \bar{\varepsilon}\langle\xi, d(t)\rangle+\langle\xi, x(t)\rangle-\bar{\varepsilon}^{3}|\xi|+\bar{\varepsilon}^{2} E(x, d \mid Q)(t, \xi)
\end{aligned}
$$

for all $(t, \xi)$ satisfying $\bar{\varepsilon}\langle\xi, d(t)\rangle>2\left[\delta^{*}(\xi \mid Q(t))-\langle\xi, x(t)\rangle\right]$. Therefore, it holds that $v \in V(x, d \mid \mathbf{Q})$.

Thus the nonemptiness of $V(x, d \mid \mathbf{Q})$ is also proved and the proof of Theorem 3.5 is complete.

Remark 3.4. When nonempty, the set $V(x, d \mid \mathbf{Q})$ is in $\boldsymbol{\Sigma}_{0}(T)$. Thus Theorems 3.2 and 3.5 and Corollary 2.3 yield that $\overline{c o} \sigma$ is the function $q(\cdot, \cdot)$ defined by (1.7). Therefore, when one of the equivalent statements of Theorem 3.5 holds, then $\overline{\mathrm{co}} \sigma$ belongs to $\Lambda_{0}(T)$; that is, in addition to its lower semicontinuity and sublinearity property, it also satisfies

$$
\overline{\mathrm{co}} \sigma(x, d \mid Q)(t, \xi)+\overline{\mathrm{co}} \sigma(x, d \mid Q)(t,-\xi)>0
$$

for all $t \in T$ and for all $\xi \in \mathbf{R}^{r}, \xi \neq 0$.

The next result is a consequence of Theorems 3.2 and 3.5. It states that the function $q(\cdot, \cdot)=\overline{\mathrm{co}} \sigma(x, d \mid Q)$, which is in $\Lambda_{0}(T)$, is the support functional corresponding via the map $(2.1)$ to the set-valued map $\bar{V}(x, d \mid \mathbf{Q})(\cdot)$, where

$$
\bar{V}(x, d \mid \mathbf{Q})(t):=\{v(t) \mid v(\cdot) \in \operatorname{cl} V(x, d \mid \mathbf{Q})\} .
$$

Corollary 3.9. The inclusion $v \in \operatorname{cl} V(x, d \mid \mathbf{Q})$ holds if and only if

$$
\langle\xi, v(t)\rangle \leq \overline{\operatorname{co}} \sigma(x, d \mid Q)(t, \xi) \quad \text { for all } \quad t \in T, \xi \in \mathbf{R}^{r} .
$$

Proof. Let $v \in \operatorname{cl} V(x, d \mid \mathbf{Q})$. Then there exists a sequence $v_{n} \in V(x, d \mid \mathbf{Q})$ such that $v_{n} \rightarrow v$. Now, by Theorem 3.2 ,

$$
\left\langle\xi, v_{n}(t)\right\rangle<\overline{\operatorname{co}} \sigma(x, d \mid Q)(t, \xi) \quad \text { for all } \quad t \in T, \xi \in\left(\mathbf{R}^{r} \backslash\{0\}\right) ;
$$

therefore, by taking the limit $n \rightarrow \infty,(3.14)$ follows.

Conversely, if $v$ satisfies (3.14), then $\sigma(x, d \mid Q)(t, \xi)>-\infty$ for all $t \in T, \xi \neq 0$. Therefore, $V(x, d \mid \mathbf{Q})$ is not empty by Theorem 3.5. Let $v_{0} \in V(x, d \mid \mathbf{Q})$ and let $v_{n}:=(1-1 / n) v+(1 / n) v_{0}$. Then $v_{n} \rightarrow v$ and (3.15) holds (by adding the inequality for $v$ and $v_{0}$ with the proper convex combination). Thus $v_{n} \in V(x, d \mid \mathbf{Q})$; whence $v \in \operatorname{cl} V(x, d \mid \mathbf{Q})$. 
In the following result, we describe how the support functional of $V(x, d \mid \mathbf{Q})$ can be evaluated in terms of $\overline{\mathrm{co}} \sigma$.

TheOREM 3.10. Assume that condition (iii) of Theorem 3.5 holds and let $\mu \in$ $\mathcal{M}(T)$ be a bounded vector-valued Borel measure on T. Then

$$
\delta^{*}(\mu \mid V(x, d \mid \mathbf{Q}))=\int_{T} \overline{\mathrm{co}} \sigma(x, d \mid Q)\left(t, \frac{d \mu}{d|\mu|}(t)\right) d|\mu|(t),
$$

where $\frac{d \mu}{d|\mu|}(\cdot)$ is the Radon-Nikodym derivative of $\mu$ with respect to $|\mu|$.

Proof. From the definition of the support functional $\delta^{*}$, the result is that

$$
\delta^{*}(\mu \mid V(x, d \mid \mathbf{Q}))=\delta^{*}(\mu \mid \operatorname{cl} V(x, d \mid \mathbf{Q})) .
$$

From Corollary 3.9 we have that

$$
q(t, \xi):=\overline{\operatorname{co}} \sigma(x, d \mid Q)(t, \xi)
$$

is the support functional on $T \times \mathbf{R}^{r}$ associated with $\operatorname{cl} V(x, d \mid \mathbf{Q})$ via the map (2.1). Thus, by Theorem 2.1, (3.16) follows.

4. Comparison with known results. In this section we specialize the statements of Theorems 3.2, 3.5, and 3.10 to the setting when $Q(\cdot)$ is the set-valued map defined by

$$
Q(t):=]-\infty, 0], \quad t \in T .
$$

We show how the function $\overline{c o} \sigma$ relates to the function $\mathbf{E}$ of Theorem 1.2 introduced by Kawasaki. The set $\mathbf{Q}$ defined in (3.1) is now the set of all nonpositive-valued continuous functions. Let $x \in \mathbf{Q}$ and $d \in T(x \mid \mathbf{Q})$ be fixed. By Theorem 2.1, the latter inclusion is equivalent to the following condition: $d$ is a continuous function satisfying

$$
d(t) \leq 0 \quad \text { if } \quad x(t)=0 \quad(t \in T) .
$$

LEMMA 4.1. Under the above assumptions,

$$
\overline{\mathrm{co}} \sigma(x, d \mid Q)(t, \xi)= \begin{cases}\xi \sigma_{x, d}(t) & \text { if } \quad t \in T, \xi>0, \\ +\infty & \text { if } \quad t \in T, \xi<0,\end{cases}
$$

where $\sigma_{x, d}$ is defined as $\sigma_{a, b}$ in Lemma 3.3 with $a=x$ and $b=d$.

Proof. Taking the definitions used in Lemma 3.4

$$
\begin{aligned}
a(t, \xi) & :=\xi x(t)-\delta^{*}(\xi \mid Q(t)), \\
b(t, \xi) & :=\xi d(t),
\end{aligned}
$$

we get that

$$
\sigma(x, d \mid Q)(t, \xi)=\sigma_{a, b}(t, \xi)=|\xi| \sigma_{x, d}(t, \operatorname{sgn}(\xi))
$$

for $t \in T$ and $\xi \neq 0$. Hence

$$
\sigma(x, d \mid Q)(t, \xi)= \begin{cases}\xi \sigma_{x, d}(t) & \text { if } \quad t \in T, \xi>0 \\ +\infty & \text { if } \quad t \in T, \xi<0\end{cases}
$$


The function on the right-hand side of this equation is sublinear in $\xi$ and lower semicontinuous in $(t, \xi)$ on $T \times(\mathbf{R} \backslash\{0\})$. Therefore, $\overline{\operatorname{co}} \sigma(x, d \mid Q)=\sigma(x, d \mid Q)$ for $t \in T$ and $\xi \neq 0$.

COROLlary 4.2. Let $\mathbf{Q}$ be the set of all nonpositive-valued continuous functions on the compact metric space $T$, and let $x \in \mathbf{Q}$ and $d \in T(x \mid \mathbf{Q})$. Then we have the following assertions:

(i) $V(x, d \mid \mathbf{Q}) \neq \emptyset$ if and only if $\sigma_{x, d}(t)>-\infty$ for all $t \in T$;

(ii) a continuous function $v: T \rightarrow \mathbf{R}$ belongs to $V(x, d \mid \mathbf{Q})$ if and only if $v(t)<$ $\sigma_{x, d}(t)$ for all $t \in T$

(iii) a continuous function $v: T \rightarrow \mathbf{R}$ belongs to $\operatorname{cl} V(x, d \mid \mathbf{Q})$ if and only if $v(t) \leq$ $\sigma_{x, d}(t)$ for all $t \in T$

(iv) if $V(x, d \mid \mathbf{Q}) \neq \emptyset$ and $\mu \in \mathcal{M}(T)$ is a nonnegative Borel measure on $T$, then

$$
\delta^{*}(\mu \mid V(x, d \mid \mathbf{Q}))=\int_{T} \sigma_{x, d}(t) d \mu(t) .
$$

Proof. Using Lemma 4.1, the statements directly follow from Theorem 3.5, Theorem 3.2, Corollary 3.9, and Theorem 3.10, respectively.

REMARK 4.1. The first and third statements of this corollary are equivalent to that of Theorem 1.2 (which is the result of Kawasaki [7], [8], since $\operatorname{cl} V(x, d \mid \mathbf{Q})=$ $T^{2}(x, d \mid \mathbf{Q})$ and $\sigma_{x, d}$ can be replaced by $-\mathbf{E}_{-x,-d}$ as we have noted in Remark 3.3.

REMARK 4.2. If the set-valued map $Q(\cdot)$ is now defined by

$$
Q(t):=]-\infty, 0]^{r} \subset \mathbf{R}^{r},
$$

then, for $x=\left(x_{1}, \ldots, x_{r}\right) \in \mathbf{Q}, d=\left(d_{1}, \ldots, d_{r}\right) \in T(x \mid \mathbf{Q})$, the results concerning the set $V(x, d \mid \mathbf{Q})$ follow from Corollary 4.2 and from the following easy-to-obtain formula

$$
V(x, d \mid \mathbf{Q})=V\left(x_{1}, d_{1} \mid \mathbf{Q}_{1}\right) \times \cdots \times V\left(x_{r}, d_{r} \mid \mathbf{Q}_{r}\right),
$$

where $\mathbf{Q}_{1}=\cdots=\mathbf{Q}_{r}$ is the set of nonpositive continuous functions.

5. Applications to optimization theory. In this section we make the following specifications of the optimization problem $(\mathcal{P})$ and Theorem 1.1. Let $T$ be a compact Hausdorff space and let $X=C\left(T, \mathbf{R}^{r}\right)$. Furthermore, let $G: D \rightarrow X$ and $\mathbf{Q} \subset X$ be given by

$$
G(z)(t)=g(t, z) \quad \text { and } \quad \mathbf{Q}:=\{x(\cdot) \mid x(t) \in Q(t) \forall t \in T\},
$$

where $g: T \times D \rightarrow \mathbf{R}^{r}$ is a continuous function and $Q: T \rightarrow 2^{\mathbf{R}^{r}}$ is a lower semicontinuous set-valued map whose values are closed convex sets with nonempty interiors. Then, by Theorem 2.2, the interior of $\mathbf{Q}$ is also nonempty. Thus $(\mathcal{P})$ reduces to the following problem:

$\left(\mathcal{P}^{*}\right) \quad$ minimize $\quad f(z) \quad$ subject to $\quad g(t, z) \in Q(t)(\forall t \in T), \quad H(z)=0$.

The main focus of this section is to apply Theorem 1.1 to the problem $\left(\mathcal{P}^{*}\right)$ in such a way that all of the hypotheses assumed and all the results obtained will be phrased explicitly in terms of the data $f, g, Q(\cdot)$, and $H$. In particular, the interpretation of the second-order necessary condition (1.3) is an important task.

- A point $\hat{z} \in D$ is admissible for $\left(\mathcal{P}^{*}\right)$ if $g(t, \hat{z}) \in Q(t)$ for all $t \in T$ and $H(\hat{z})=0$. 
- The regularity of $\hat{z}$ means that the assumptions (R1) and (R3) are valid and, in addition,

$\left(\mathrm{R} 2^{*}\right)$ the map $g(t, \cdot)$ is uniformly strictly Fréchet differentiable at $\hat{z}$ for all $t \in T$; that is,

$$
\lim _{z_{1}, z_{2} \rightarrow \hat{z}} \frac{\left|g\left(t, z_{1}\right)-g\left(t, z_{2}\right)-g^{\prime}(t, \hat{z})\left(z_{1}-z_{2}\right)\right|}{\left\|z_{1}-z_{2}\right\|}=0
$$

holds uniformly for $t \in T$.

Then with the definition (5.1) the map $G$ is strictly Fréchet differentiable at $\hat{z}$; therefore, (R2) holds too.

- A vector $d$ is critical for $\left(\mathcal{P}^{*}\right)$ at an admissible regular point $\hat{z}$ if $(\mathrm{C} 1)$, (C3) are valid; furthermore,

$\left(\mathrm{C} 2^{*}\right) g^{\prime}(t, \hat{z}) d \in T(g(t, \hat{z}) \mid Q(t))$ for all $t \in T$.

This assumption, by Theorem 2.1, is equivalent to (C2).

- The vector $d$ is a regular direction if (R4) holds,

$\left(\mathrm{R} 5^{*}\right)$ the following second-order directional derivative exists for all $t \in T$ :

$$
g^{\prime \prime}(t, \hat{z}, d):=\lim _{\varepsilon \rightarrow 0+} 2 \frac{g(t, \hat{z}+\varepsilon d)-g(t, \hat{z})-\varepsilon g^{\prime}(t, \hat{z}) d}{\varepsilon^{2}},
$$

and the limit is uniform in $t$; furthermore, $H$ satisfies the same assumption as in (R5).

$\left(\mathrm{R} 6^{*}\right) \sigma\left(g(\cdot, \hat{z}), g^{\prime}(\cdot, \hat{z}) d(\cdot) \mid Q(\cdot)\right)(t, \xi)>-\infty$ for $t \in T, \xi \in \mathbf{R}^{r}$, where the function $\sigma(x, d \mid Q(\cdot))=\sigma(x, d \mid Q)$ is defined in (3.3).

It is easy to see that $\left(\mathrm{R} 5^{*}\right)$ yields that the map $G$ defined in (5.1) satisfies the condition (R5). The regularity assumption made in $\left(\mathrm{R} 6^{*}\right)$ is also equivalent to (R6) by Theorem 3.5 .

After these considerations, the main result of the section is the following theorem. Its proof employs the results derived in section 3 .

THEOREM 5.1. Let $\hat{z}$ be a regular solution of the above problem $\left(\mathcal{P}^{*}\right)$. Then, for all regular critical directions $d$, there exist Lagrange multipliers $\lambda \geq 0$, a signed vector-valued measure $\mu \in \mathcal{M}\left(T, \mathbf{R}^{r}\right)$, and $y^{*} \in Y^{*}$ such that at least one of them is different from zero and the following relations hold:

$$
\lambda f^{o}(\hat{z} ; z)+\int_{T} g^{\prime}(t, \hat{z}) z d \mu(t)+\left\langle y^{*}, H^{\prime}(\hat{z}) z\right\rangle \geq 0 \quad \text { for } \quad z \in Z
$$

and

$$
\begin{aligned}
\lambda f^{o o}(\hat{z}, d)+ & \int_{T} g^{\prime \prime}(t, \hat{z}, d) d \mu(t)+\left\langle y^{*}, H^{\prime \prime}(\hat{z}, d)\right\rangle \\
& \geq 2 \int_{T} \overline{\operatorname{co}} \sigma\left(g(\cdot, \hat{z}), g^{\prime}(\cdot, \hat{z}) d \mid Q\right)\left(t, \frac{d \mu}{d|\mu|}(t)\right) d|\mu|(t)
\end{aligned}
$$

Proof. As we have already verified, the assumptions of Theorem 1.1 follow from that of Theorem 5.1 if we define $G$ and $\mathbf{Q}$ by (5.1). Thus it suffices to show that the conclusions of Theorem 1.1 yield that of Theorem 5.1. 
The existence of the Lagrange multiplier $x^{*} \in X^{*}=\left(C\left(T, \mathbf{R}^{r}\right)\right)^{*}$ is equivalent to the existence of the measure $\mu \in \mathcal{M}\left(T, \mathbf{R}^{r}\right)$ by Riesz's representation theorem. Applying Theorems 2.1 and 3.10, (5.2), (5.3), and (5.4) are equivalent to (1.1), (1.2), and (1.3), respectively.

We consider now a special case of Theorem 5.1 when the compact metric space consists of a single element:

$\left(\mathcal{P}^{* *}\right) \quad$ minimize $\quad f(z)$ subject to $\quad g(z) \in Q, \quad H(z)=0$.

Here $Q \subset \mathbf{R}^{r}$ is a closed convex set with nonempty interior and $g: D \rightarrow \mathbf{R}^{r}$ is a continuous function on $D$. The assumptions $\left(R 2^{*}\right),\left(C 2^{*}\right),\left(R 5^{*}\right)$, and $\left(R 6^{*}\right)$ of problem $\left(\mathcal{P}^{*}\right)$ have to be replaced by

$\left(\mathrm{R} 2^{* *}\right)$ The map $g(\cdot)$ is strictly Fréchet differentiable at $\hat{z}$;

$\left(\mathrm{C} 2^{* *}\right) g^{\prime}(\hat{z}) d \in T(g(\hat{z}) \mid Q)$

$\left(\mathrm{R} 5^{* *}\right)$ the following second-order directional derivative exists

$$
g^{\prime \prime}(\hat{z}, d):=\lim _{\varepsilon \rightarrow 0+} 2 \frac{g(\hat{z}+\varepsilon d)-g(\hat{z})-\varepsilon g^{\prime}(\hat{z}) d}{\varepsilon^{2}} ;
$$

$\left.\left(\mathrm{R} 6^{* *}\right) \sigma\left(g(\hat{z}), g^{\prime}(\hat{z}) d \mid Q\right)\right)(\xi)>-\infty$ for $\xi \in \mathbf{R}^{r}$.

Corollary 5.2. Let $\hat{z}$ be a regular solution of the above problem $\left(\mathcal{P}^{* *}\right)$. Then, for all regular critical directions $d$, there exist Lagrange multipliers $\lambda \geq 0$, a vector $\mu \in \mathbf{R}^{r}$, and $y^{*} \in Y^{*}$ such that at least one of them is different from zero and the following relations hold:

$$
\lambda f^{o}(\hat{z} ; z)+\mu^{T} g^{\prime}(\hat{z}) z+\left\langle y^{*}, H^{\prime}(\hat{z}) z\right\rangle \geq 0 \quad \text { for } \quad z \in Z
$$

and

$$
\lambda f^{o o}(\hat{z}, d)+\mu^{T} g^{\prime \prime}(\hat{z}, d)+\left\langle y^{*}, H^{\prime \prime}(\hat{z}, d)\right\rangle \geq 2 \overline{\operatorname{co}} \sigma\left(g(\hat{z}), g^{\prime}(\hat{z}) d \mid Q\right)(\mu) .
$$

Proof. Let $T=\{0\}$ and $\tilde{g}: T \times D \rightarrow \mathbf{R}^{r}$ be defined by $\tilde{g}(t, z):=g(z)$, and let $\tilde{Q}(\cdot): T \rightarrow 2^{\mathbf{R}^{r}}$ be given by $\tilde{Q}(t)=Q$. Making these substitutions, problem $\left(\mathcal{P}^{* *}\right)$ reduces to $\left(\mathcal{P}^{*}\right)$, where $g$ and $Q(\cdot)$ are replaced by $\tilde{g}$ and $\tilde{Q}(\cdot)$, respectively. The assumptions indicated by ${ }^{* *}$ yield the analogous ones marked by ${ }^{*}$. Thus the conclusion of Theorem 5.1 is valid. Observe that, in this case, a vector-valued measure on $T$ can be identified with a single vector in $\mathbf{R}^{r}$. Thus the conclusion of Theorem 5.1 reduces to that of Corollary 5.2.

We consider now another special case of Theorem 5.1, when $Q(t)=]-\infty, 0]$ for all $t \in T$. That is we investigate the following problem:

$\left(\mathcal{P}^{* * *}\right) \quad$ minimize $\quad f(z)$ subject to $\quad g(t, z) \leq 0(\forall t \in T) \quad H(z)=0$.

Here $g: T \times D \rightarrow \mathbf{R}$ is a continuous function. The assumptions $\left(C 2^{*}\right)$ and $\left(R 6^{*}\right)$ of problem $\left(\mathcal{P}^{*}\right)$ have to be replaced by

$\left(\mathrm{C} 2^{* * *}\right) g^{\prime}(t, \hat{z}) d(t) \leq 0$ if $g(t, \hat{z})=0$

$\left(\mathrm{R} 6^{* * *}\right) \sigma_{g(\cdot, \hat{z}), g^{\prime}(\cdot, \hat{z}) d(\cdot)}(t)>-\infty$ for $t \in T$. 
Corollary 5.3. Let $\hat{z}$ be a regular solution of the above problem $\left(\mathcal{P}^{* * *}\right)$. Then, for all regular critical directions $d$, there exist Lagrange multipliers $\lambda \geq 0$, a nonnegative (scalar-valued) measure $\mu \in \mathcal{M}(T, \mathbf{R})$, and $y^{*} \in Y^{*}$ such that at least one of them is different from zero and the following relations hold:

$$
\begin{gathered}
\operatorname{supp} \mu \subset\{t \in T \mid g(t, \hat{z})=0\}, \\
\lambda f^{o}(\hat{z} ; z)+\int_{T} g^{\prime}(t, \hat{z}) z d \mu(t)+\left\langle y^{*}, H^{\prime}(\hat{z}) z\right\rangle \geq 0 \quad \text { for } \quad z \in Z,
\end{gathered}
$$

and

$$
\lambda f^{o o}(\hat{z}, d)+\int_{T} g^{\prime \prime}(t, \hat{z}, d) d \mu(t)+\left\langle y^{*}, H^{\prime \prime}(\hat{z}, d)\right\rangle \geq 2 \int_{T} \sigma_{g(\cdot, \hat{z}), g^{\prime}(\cdot, \hat{z}) d(\cdot)}(t) d \mu(t) .
$$

Proof. The equivalence of conditions (5.4) and (5.10) follows from Lemma 4.1 and Corollary 4.2. Condition (5.2) can be translated into the following form:

$$
\frac{d \mu}{d|\mu|}(t) \begin{cases}\geq 0 & \text { if } \quad g(t, \hat{z})=0 \\ =0 & \text { if } \quad g(t, \hat{z})<0 .\end{cases}
$$

Therefore, $\mu$ is nonnegative valued and (5.8) holds.

To conclude this section, we present a numerical example illustrating how the function $\sigma$ can be explicitly calculated. The problem considered in the example can be handled with classical results as well. Our intention is to demonstrate how our results provide a new method for computing a second-order optimality criterion that does not require the computation of the set of the second-order tangent variations. Instead, we only need to calculate the function $\sigma$. For problems that cannot be handled without using the envelope-effect theory, we refer the readers to the papers of Kawasaki [6], [7], [8].

Example. Let $Q$ be the closed unit disk in $\mathbf{R}^{2}$ and $f\left(z_{1}, z_{2}\right):=-z_{2}^{2}-2 z_{1}$ for $\left(z_{1}, z_{2}\right) \in \mathbf{R}^{2}$. We consider the following optimization problem: minimize $f$ on $Q$. As we shall see, the point $\hat{z}=(1,0)$ satisfies the first- and second-order necessary conditions of Corollary 5.2. (Here we take the function $g=\mathrm{id}$ and the vector space $Y=\{0\}$ and $H=0$.)

It is easy to see that $\hat{z}$ is an admissible regular point for this problem. A vector $d=\left(d_{1}, d_{2}\right)$ is a critical direction if

$$
f^{\prime}(\hat{z}) d \leq 0 \quad \text { and } \quad d \in T(\hat{z} \mid Q),
$$

that is, if $d_{1}=0$. Therefore, $d=(0,1)$ is a (typical) critical direction. We show that it is also regular. We have to check condition $\left(R 6^{* *}\right)$. Observe that

$$
\mathcal{C}(\hat{z}, d \mid Q)=\left\{\left(\xi_{1}, \xi_{2}\right) \mid \xi_{1} \geq 0, \xi_{2}=0\right\}
$$

as it is defined in (3.2) (we simply omit $t, T$ from the definitions, because they do not play any role). On the other hand, if $\xi=\left(\xi_{1}, \xi_{2}\right)$, then $\delta^{*}(\xi \mid Q)=|\xi|$. Thus, for $\mathcal{S}(\hat{z}, d \mid Q)$, we have

$$
\mathcal{S}(\hat{z}, d \mid Q)=\left\{\left(\xi_{1}, \xi_{2}\right) \mid \xi_{2}>0\right\}
$$


Clearly, $\mathcal{C}(\hat{z}, d \mid Q) \subset \mathcal{S}^{\prime}(\hat{z}, d \mid Q)$. Therefore, if $\xi=\left(\xi_{1}, 0\right) \in \mathcal{C}(\hat{z}, d \mid Q)$, then

$$
\begin{aligned}
\sigma(\hat{z}, d \mid Q)(\xi) & =\liminf _{\substack{\left(\zeta_{1}, \zeta_{2}\right) \rightarrow\left(\xi_{1}, 0\right) \\
\zeta_{2}>0}} \frac{\zeta_{2}^{2}}{4\left[\zeta_{1}-\sqrt{\zeta_{1}^{2}+\zeta_{2}^{2}}\right]} \\
& =\liminf _{\substack{\left(\zeta_{1}, \zeta_{2}\right) \rightarrow\left(\xi_{1}, 0\right) \\
\zeta_{2}>0}} \frac{\zeta_{2}^{2}\left(\zeta_{1}+\sqrt{\zeta_{1}^{2}+\zeta_{2}^{2}}\right)}{-4 \zeta_{2}^{2}}=\frac{-\xi_{1}}{2}>-\infty,
\end{aligned}
$$

and $\sigma(\hat{z}, d \mid Q)=+\infty$ otherwise. We have checked that $\left(R 6^{* *}\right)$ holds, too. Furthermore, we also see that $\sigma(\hat{z}, d \mid Q)=\overline{\operatorname{co}} \sigma(\hat{z}, d \mid Q)$ in this case.

To complete this argument, we have to show that there exist $\lambda \geq 0$ and $\mu=$ $\left(\mu_{1}, \mu_{2}\right)$ such that (5.5), (5.6), and (5.7) hold. It follows from (5.6) that $\mu_{2}=0$ and $\mu_{1} \geq 0$. The inequality in (5.6) is satisfied if and only if

$$
\lambda \partial_{1} f(\hat{z})+\mu_{1}=-2 \lambda+\mu_{1}=0 \quad \text { and } \quad \lambda \partial_{2} f(\hat{z})=0 .
$$

If $\lambda$ were 0 , then, by the first equation, $\mu_{1}=0$, which contradicts $\lambda+|\mu| \neq 0$. Thus we may assume that $\lambda=1$. Then $\mu_{1}=2$. We have to check whether (5.5) is also satisfied. For the right-hand side of $(5.7)$ we have $2 \sigma(\hat{z}, d \mid Q)(2,0)=-2$. On the other hand,

$$
f^{o o}(\hat{z})(d)=d^{T} f^{\prime \prime}(\hat{z}) d=\partial_{2}^{2} f(\hat{z})=-2,
$$

which shows that (5.7) is satisfied by equality.

It is also easy to see that $f$ attains minimum on $Q$ at $\hat{z}=(0,1)$, because, for $|z| \leq 1$

$$
f(z)=-z_{2}^{2}-2 z_{1} \geq-1+z_{1}^{2}-2 z_{1}=\left(z_{1}-1\right)^{2}-2 \geq-2=f(1,0) .
$$

\section{REFERENCES}

[1] F. H. Clarke, Optimization and Nonsmooth Analysis, Canadian Math. Soc. Ser. Monogr. Adv. Texts, John Wiley, New York, 1983.

[2] R. Cominetti, Metric regularity, tangent sets and second-order optimality conditions, Appl. Math. Optim., 21 (1990), pp. 265-287.

[3] A. Ya. Dubovitski and A. A. Milyutin, Extremum problems with constraints, Dokl. Akad. Nauk SSSR, 149 (1963), pp. 759-762; Soviet Math. Dokl., 4 (1963), pp. 452-455.

[4] I. V. Girsanov, Lectures on Mathematical Theory of Extremum Problems, Lecture Notes in Econom. and Math. Systems 67, Springer-Verlag, New York, 1972.

[5] A. D. IofFE, On some recent developments in the theory of second-order optimality conditions, in Optimization, S. Dolecki, ed., Fifth French-German Conference Castel-Novel, 1988. Lecture Notes in Math. 1405, Springer-Verlag, New York, 1989, pp. 55-68.

[6] H. KAWASAKI, An envelope-like effect of infinitely many inequality constraints on second-order necessary conditions for minimization problems, Math. Programming, 41 (1988), pp. 7396.

[7] H. KAWASAKI, The upper and lower second order directional derivatives of a sup-type function, Math. Programming, 41 (1988), pp. 327-339.

[8] H. KaWASAKI, Second order necessary optimality for minimizing a sup-type function, Math. Programming, 49 (1991), pp. 213-229.

[9] Y. Maruyama, Second-order necessary conditions for nonlinear optimization problems in Banach spaces by the use of Neustadt derivative, Math. Japonica, 40 (1994), pp. 509-522.

[10] P. D. Loewen and R. T. Rockafellar, The adjoint arc in nonsmooth optimization, Trans. Amer. Math. Soc., 325 (1991), pp. 39-72.

[11] Zs. PÁles AND V. M. ZeIdAn, Nonsmooth optimum problems with constraints, SIAM J. Control Optim., 32 (1994), pp. 1476-1502.

[12] Zs. PÁles And V. M. Zeidan, Characterization of closed $C$-convex sets in $C\left(T, \mathbf{R}^{n}\right)$, Acta Sci. Math. Szeged, submitted. 
[13] J.-P. Penot, Optimality conditions in mathematical programming and composite optimization, Mathematical Programming 67 (1994), pp. 225-245.

[14] R. T. Rockafellar, Convex Analysis, Princeton University Press, Princeton, NJ, 1970.

[15] R. T. Rockafellar, Integrals which are convex functionals, Pacific J. Math., 24 (1968), pp. 525-539.

[16] R. T. Rockafellar, Integrals which are convex functionals, II, Pacific J. Math., 39 (1971), pp. 439-469. 